\title{
Transversity Measurements at COMPASS
}

\author{
C. Schill on behalf of the COMPASS Collaboration \\ Physikalisches Institut der Universität Freiburg \\ Hermann-Herder Str. 3, D-79104 Freiburg, Germany.
}

\begin{abstract}
The measurement of transverse spin effects in semi-inclusive deep-inelastic scattering is an important part of the COMPASS physics program. From the analysis of the 2002-2004 data, new results for the transverse target spin asymmetry of z-ordered identified pion and kaon pairs are presented [1. In addition, a first result for the transverse target spin asymmetry of exclusively produced $\rho^{0}$ mesons on the deuteron is shown.
\end{abstract}

\section{Introduction}

Single spin asymmetries in semi-inclusive deep-inelastic scattering off transversely polarized nucleon targets have been under intense experimental and theoretical investigation over the past few years 2, 3, 4, They provide new insights into QCD and the nucleon structure. For instance, they allow the determination of the third yetunknown leading-twist quark distribution function $\Delta_{T} q(x)$, the transversity distribution 5, 6. Additionally, they give insight into the parton transverse momentum distribution and angular momentum 7 .

COMPASS exploited three different "quark polarimeters" to access transversity: For hadron production in deep-inelastic scattering the azimuthal asymmetry of the produced hadrons was measured. This asymmetry gives access to transversity by the Collins mechanism. Another probe to access transversity is the interference fragmentation function in the production of hadron pairs [8, 9]. Finally, for the production of $\Lambda$ baryons the transverse spin of quarks is transferred to the transverse lambda polarization, which is determined experimentally.

In hard exclusive production of $\rho^{0}$ mesons on a transversely polarized target, a different aspect of the spin structure of the nucleon can be probed. Since few years it is well established that hard meson production is a very good candidate to study the universal generalized parton distributions (GPDs) 10, 11, 12. These distributions describe in the most complete way the nucleon structure and encode fundamental information in particular about the angular momentum carried by partons and about their spatial distribution.

At the COMPASS experiment at CERN all channels mentioned above have been studied. The following part will focus on two new results, the Collins asymmetry for identified z-ordered hadron pairs and the target spin asymmetry for exclusively produced $\rho^{0}$ mesons.

\section{Two-hadron asymmetry}

At leading twist, the fragmentation function $(F F)$ of a polarized quark into a pair of hadrons is expected to be of the form

$F F=D_{q}^{2 h}\left(z, M_{h}^{2}\right)+H_{1}^{\varangle}\left(z, M_{h}^{2}\right) \sin \theta \sin \phi_{R S}$,

where $M_{h}$ is the invariant mass of the hadron pair and $z=z_{1}+z_{2}$ is the fraction of available energy carried by the two hadrons. $D_{q}^{2 h}\left(z, M_{h}^{2}\right)$ is the unpolarized fragmentation function into two hadrons. The angles $\theta$ and $\phi_{R S}$ are defined according to Ref. [13. $\phi_{R S}=\phi_{R}+\phi_{S}-\pi$ is the sum of the azimuthal angle $\phi_{R}$ of a plane containing the two hadrons and the azimuthal angle $\phi_{S}$ of 
target spin vector with respect to the lepton scattering plane. $\theta$ is the polar angle of the first hadron in the two-hadron center-of-mass frame with respect to the direction of the summed hadron momentum $\vec{p}_{h}=\vec{p}_{1}+\vec{p}_{2}$. Within the COMPASS acceptance, $\theta$ peaks close to $\pi / 2$ with $\langle\sin \theta>\approx 0.95$. The following results are obtained by integrating over $\sin \theta$. The number of hadron pairs in a bin of $x, z$ or $M_{h}$ is given by

$$
N^{ \pm}\left(\phi_{R S}\right)=N_{0} \cdot\left(1 \pm A_{U T}^{\sin \phi_{R S}} \cdot \sin \phi_{R S}\right),
$$

where \pm refers to the transverse target spin orientation and $N_{0}$ is the mean number of detected hadron pairs averaged over $\sin \phi_{R S}$. From the angular distribution of the hadron pairs, one can thus measure the asymmetry

$$
A_{R S}=\frac{1}{f P_{T} D} \cdot A_{U T}^{\sin \phi_{R S}},
$$

where $f \approx 0.38$ is the target dilution factor, $P_{T}$ the target polarization and $D$ the depolarization factor given by $D=(1-y) /(1-$ $\left.y+y^{2} / 2\right)$, where $y$ is the fractional energy transfer of the lepton.

The measured asymmetry can be factorized into a convolution of the transversity distribution $\Delta_{T} q(x)$ of the quarks of flavor $q$ and the interference fragmentation $H_{1}^{\varangle}\left(z, M_{h}^{2}\right)$ 13]:

$$
A_{R S}=\frac{\Sigma_{q} e_{q}^{2} \cdot \Delta_{T} q(x) \cdot H_{1}^{\varangle}\left(z, M_{h}^{2}\right)}{\Sigma_{q} e_{q}^{2} \cdot q(x) \cdot D_{q}^{2 h}\left(z, M_{h}^{2}\right)},
$$

summed over all quark flavors $q$.

\section{Event selection}

The data discussed here have been taken in the years 2003 to 2004 at the COMPASS experiment at CERN. It scatters a $160 \mathrm{GeV}$ $\mu^{+}$beam on a transversely polarized solid state ${ }^{6} \mathrm{LiD}$ target. The scattered muons and the produced hadrons are detected in a $50 \mathrm{~m}$ long large-acceptance forward spectrometer with excellent particle identification capabilities. A large scale Ring Imaging Cherenkov
Detector (RICH) is used to distinguish pions, kaons and protons [14.

The event selection was done in the same way as in the previous analysis of the Collins and Sivers asymmetries for single hadrons 2. For the selection of the DIS event sample, kinematic cuts of the squared fourmomentum transfer $Q^{2}>1(\mathrm{GeV} / \mathrm{c})^{2}$, the hadronic invariant mass $W>5 \mathrm{GeV} / \mathrm{c}^{2}$ and the fractional energy transfer of the muon $0.1<y<0.9$ were applied.

Hadron pairs originating from the primary vertex are selected. The hadrons are separated into $\pi$ and $K$ pairs. A selection cut of $z_{1 / 2}>0.1$ suppresses hadrons from the target fragmentation. The two leading hadrons have been selected ordered according to their fractional energy, taking the first hadron as the most energetic one. A cut on the sum $z=z_{1}+z_{2}<0.9$ rejects exclusively produced $\rho$ and $\phi$ mesons.

By combining data from both target cells as well as from sub-periods with opposite target polarization in a double ratio product described in detail in Ref. [2, the acceptance function of the spectrometer cancels out and the azimuthal asymmetry $A_{R S}\left(x, z, M_{h}\right)$ is extracted by a fit to the data. In various studies, it was shown that systematic effects of the measurement are considerably smaller than the statistical uncertainty of the data.

\section{Results}

In figure 1 the results for the target single spin asymmetry $A_{R S}$ for identified leading zordered $\pi \pi$ and $K \pi$ pairs are shown. The asymmetries are plotted as a function of the hadron pair invariant mass $M_{i n v}$. The measured asymmetries are small and compatible with zero within the statistical precision of the data points. They do not show a significant dependence on the kinematic variables $M_{i n v}$, or $x$ and $z$, respectively. 

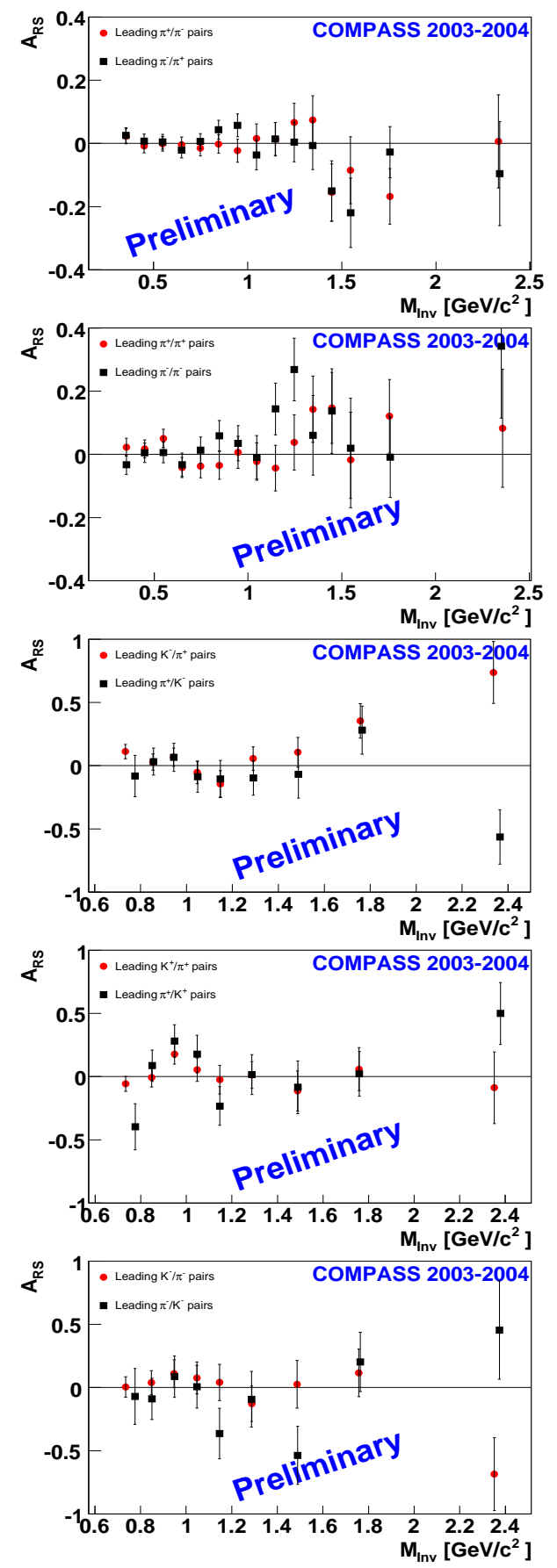

Figure 1: Asymmetries $A_{R S}$ for identified leading $\pi \pi$ pairs with opposite (top) and equal (second) charge, for identified $K \pi$ pairs with opposite (third) and equal pos. (fourth) and neg. charge (bottom panel) versus $M_{i n v}$.

\section{Discussion}

In several theoretical models, predictions have been made for the measured asymmetries $A_{R S}\left(x, z, M_{h}\right)$ for pions or unidentified hadrons on a deuteron target [4, 8]. The expected values of the asymmetry are generally small and below $1 \%$. The small signal is attributed in these calculations to a partial cancellation of the asymmetries originating from scattering on up and down quarks of the proton and neutron in the isoscalar deuteron target. In 2007, COMPASS is taking data with a transversely polarized proton target, where the asymmetries are expected to be larger 3,4. Together with the deuteron data presented here, a separation of the asymmetries originating from up and down quarks shall then become possible.

\section{Exclusive $\rho^{0}$ production}

Hard meson production has been shown to be one possible way to access generalized parton distributions. Here we have studied the exclusive production of $\rho^{0}$ mesons on a transversely polarized deuteron target [15].

The analysis is based on the complete COMPASS transverse data set from 20022004. For the selection of DIS events, the same cuts as described in section 3 have been applied. To select exclusive $\rho^{0}$, a missing energy cut of $-2.5 \mathrm{GeV}<E_{\text {miss }}<2.5 \mathrm{GeV}$ has been applied. $\rho^{0}$ mesons have been selected in a invariant mass window of $300 \mathrm{MeV}$ around the $\rho^{0}$ mass. A cut on the squared transverse momentum $p_{t}^{2}$ of the $\rho^{0}$ meson in the range between 0.01 and $0.5 \mathrm{GeV} / \mathrm{c}^{2}$ has been applied, where the lower limit ensures an accurate measurement of the azimuthal angle and the upper one suppresses non-exclusive background. In total $270 k \rho^{0}$ mesons have been reconstructed. The selected data sample contains $\rho^{0}$ produced incoherently and coherently on the deuteron target as well.

An azimuthal asymmetry $A^{\sin \eta}$ has been 

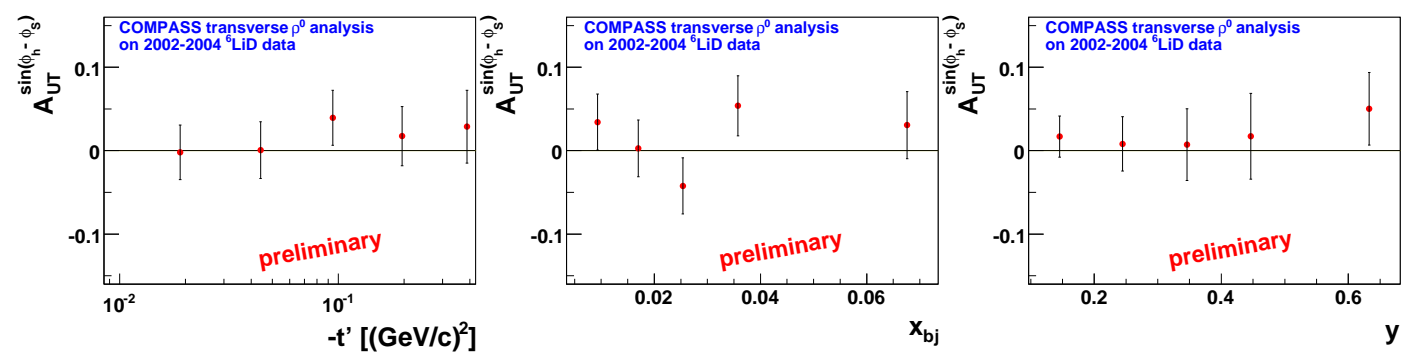

Figure 2: Target single spin asymmetry $A_{U T}$ for exclusive $\rho^{0}$ production on the deuteron.

determined with respect to the angle $\eta=$ $\phi_{\rho}-\phi_{S}$, where $\phi_{\rho}$ is the azimuthal angle of the $\rho^{0}$ meson with respect to the lepton scattering plane. The transverse target spin asymmetry has been calculated from the measured asymmetry $A^{\sin \eta}$

$$
A_{U T}^{\sin \eta}=\frac{1}{f \cdot P_{T}} A^{\sin \eta}
$$

where $f=0.38$ is the dilution factor and $P_{T}$ the mean target polarization.

Results for the asymmetry $A_{U T}$ as a function of the transferred four momentum above threshold $t^{\prime}$, of $x$ and of the fractional photon energy transfer $y$ are shown in figure 2

\section{$7 \quad$ Discussion of $\rho^{0}$ results}

For longitudinal virtual photons, factorization of the exclusive vector meson production cross section into a hard part sensitive to GPDs and a fragmentation function has been shown [16. The cross sections for transversely polarized photons was shown to be suppressed by $1 / Q^{2}$ compared to the longitudinal one. For the COMPASS kinematics in this analysis, the mean $\left\langle Q^{2}>\approx 2 \mathrm{GeV}\right.$, which gives a ratio of $\sigma_{L} / \sigma_{T} \approx 1$. Further studies will aim in a separation of the longitudinal and transverse part of the cross section using the angular distribution of the $\rho^{0}$ decay products 12 .

The current data taking at COMPASS with a transversely polarized $\mathrm{NH}_{3}$ target will provide the possibility to study exclusive meson production on the proton as well.

\section{References}

[1] Slides:

http://indico.cern.ch/materialDisplay.py? contribId=6\&session Id $=34$ \&materialld=slides\&conf $I d=3841$

[2] V.Yu. Alexakhin et al. [COMPASS collaboration] Phys. Rev. Lett. 94, 202002 (2005) and E.S. Ageev et al. [COMPASS collaboration] Nucl. Phys. B765, 31 (2007), and references therein.

[3] A. Airpetian et al. [HERMES Collaboration], Phys. Rev. Lett. 94, 012002 (2005).

[4] A. Bacchetta and M. Radici, Phys. Rev. D67, 094002 (2003), Phys. Rev. D69, 074026 (2004) and Phys. Rev. D74, 114007 (2006).

[5] J.C. Collins et al., Nucl. Phys. B420, 565 (1994).

[6] X. Artru and J.C. Collins, Z. Phys. C69, 277 (1996).

[7] R.L. Jaffe et al., Phys. Rev. Lett. 80, 1166 (1998).

[8] A. Bianconi et al., Phys. Rev. D62, 034008 (2000).

[9] M. Radici et al., Phys. Rev. Lett D65,074031 (2002).

[10] M. Vanderhaegen et al., Phys. Rev. D60, 094017 (1999).

[11] K. Goeke et al., Prog. Part. in Nucl. Phys. 47, 401 (2001).

[12] M. Diehl et al., Eur. Phys. J C41, 515 (2005).

[13] X. Artru, hep-ph/0207309 (2002).

[14] P. Abbon et al. [COMPASS collaboration], NIM A577, 455-518 (2007).

[15] J. Kiefer, Diploma Thesis, University of Freiburg (Aug. 2007), http://hadron.physik.unifreiburg.de/arbeiten/diplomarbeiten/kiefer.ps

[16] J.C. Collins et al., Phys. Rev. D55, 7114 (1997). 